P. Ponomarev

Nagoya Math. J.

Vol. 81 (1981), 123-151

\title{
TERNARY QUADRATIC FORMS AND SHIMURA'S CORRESPONDENCE
}

\author{
PAUL PONOMAREV*
}

\section{Introduction}

In his paper [11] Shimura defined a correspondence between modular forms of half integral weight and modular forms of integral weight. To each pair $(t, f(z))$, consisting of a square-free integer $t \geq 1$ and a cusp form $f(z)=\sum_{n=1}^{\infty} a(n) e^{2 \pi i n z}$ of weight $\kappa / 2$ ( $\kappa$ odd, $\geq 3$ ), level $N$ (divisible by 4 ) and character $\chi$, he associated a certain function $f^{(t)}(z)\left(F_{t}(z)\right.$ in Shimura's notation). He showed that $f^{(t)}$ was an integral modular form of weight $\kappa-1$ and character $\chi^{2}$, provided $f$ was a common eigenfunction of the Hecke operator $T_{\kappa, x}^{N}\left(p^{2}\right)$ for certain primes $p \mid N$. Furthermore, he showed that $f^{(t)}$ was a cusp form if $\kappa \geq 5$. He conjectured that the level of $f^{(t)}$ could be taken as $N / 2$. Niwa [6] verified this conjecture for $\kappa \geq 7$ by constructing a kernel function for the "lifting" $f \mapsto f^{(1)}$. In particular his approach obviates any assumption on eigenfunctions. The dependence on $t$ of the correspondence $f \mapsto f^{(t)}$ can be circumvented if $f$ is a common eigenfunction for all $T_{x, x}^{N}\left(p^{2}\right)$. In that case Shimura showed that $f^{(t)}(z)=$ $a(t) F(z)$, where $F$ is independent of $t$. This allows one to define a "lifting" $f \mapsto F$ which we call the Shimura lifting.

In this paper we determine the effect of Shimura's correspondence on the theta series associated to certain positive definite ternary quadratic forms. These quadratic forms are associated to certain maximal lattices and their duals (see $\S 1$ ). Let $L$ be one of these lattices, with ambient space $V$, and let $\theta=\theta(z, L)$ be the associated theta series. In $\S 5$ we express $\theta^{(t)}$ as an explicit linear combination of theta series associated to maximal lattices from a quaternion algebra $\mathfrak{A}$ which is naturally paired with $V$. Our approach is purely arithmetic. It is based on the fundamental idea of Eichler ([1], Chapter IV) that the effect of a Hecke operator on the

Received August 22, 1979.

*) Partially supported by NSF grant MCS 76-08746 A01. 
theta series coming from a similitude genus ("Idealkomplex" in Eichler's terminology) is the same as a certain "Anzahlmatrix" which is defined in a purely arithmetic way. Following Rallis [10], we call this "Eichler's commutation relation." In $\S 4$ we show that the Anzahlmatrices associated to the lattices $L$ are "reduced" Brandt matrices associated to a quaternion algebra $\mathfrak{A}$. This, combined with the commutation relations, allows us to explicitly determine $\theta^{(t)}$. The same approach can be used to evaluate the effect of the Doi-Naganuma lifting on theta series. The details will appear elsewhere.

\section{§1. Preliminaries}

Let $V$ be a nondegenerate quadratic space of dimension $n$ over $\boldsymbol{Q}$ and $q: V \rightarrow \boldsymbol{Q}$ the quadratic mapping on $V$. The symmetric bilinear form $B$ associated to $q$ is given by:

$$
B(v, w)=q(v+w)-q(v)-q(w) \quad \text { for } v, w \in V .
$$

$A$ similitude of $V$ is an element $\sigma \in G L(V)$ such that $q(\sigma(v))=a_{\sigma} q(v)$ for every $v \in V$, where $a_{\sigma} \in \boldsymbol{Q}^{\times}$is independent of $v$. We call $a_{\sigma}$ the norm of $\sigma$ and denote it by $n(\sigma)$. If $\operatorname{dim} V$ is odd, then $r \in Q^{\times}$is the norm of a similitude $\Leftrightarrow r$ is a square. An orthogonal transformation is a similitude of norm 1 .

By a lattice on $V$ we will mean $a Z$-lattice in $V$ of rank $n$. The discriminant $\Delta(L)$ of a lattice $L$ on $V$ is defined by:

$$
\Delta(L)=\operatorname{det}\left[B\left(v_{i}, v_{j}\right)\right],
$$

where $\left\{v_{i}\right\}$ is a $Z$-basis of $L$. The norm of $L$ is the positive rational number $n(L)$ which generates the $Z$-span of all $q(v), v \in L$. The reduced discriminant $\Delta^{\prime}(L)$ is defined by:

$$
\Delta^{\prime}(L)=\operatorname{det}\left[n(L)^{-1} B\left(v_{i}, v_{j}\right)\right],
$$

where $\left\{v_{i}\right\}$ is a $Z$-basis of $L$. It is clear that $\Delta^{\prime}(L) \in Z$. A lattice $L$ on $V$ which is maximal among all lattices on $V$ of norm $r$ is called $r$-maximal.

The notions of discriminant, norm and reduced discriminant all carry over in the obvious way to $Z_{p}$-lattices on $V_{p}=V \otimes_{\boldsymbol{Q}} \boldsymbol{Q}_{p}, p$ a rational prime. Depending on the situation, they may be regarded either as fractional ideals of $\boldsymbol{Q}_{p}$ or powers of the prime $p$. For a lattice $L$ on $V$ we set $L_{p}=L \otimes_{Z} Z_{p}$. Two lattices $L, M$ on $V$ are said to be similar, or in 
the same similitude class, if $\sigma(L)=M$ for some similitude $\sigma$ of $V$; they are in the same similitude genus ("Idealkomplex" in the sense of Eichler [1]) if $L_{p}, M_{p}$ are similar for all $p$. It is well known that the number of similitude classes in a similitude genus is finite ([1], p. 79).

An integral quadratic form in $n$ variables is a homogeneous polynomial $f=f\left(X_{1}, \cdots, X_{n}\right)$ of degree two with coefficients in $Z$. The discriminant of $f$ is taken to be the determinant $\Delta=\Delta(f)$ of the matrix

$$
A=\left[\frac{\partial^{2} f}{\partial X_{i} \partial X_{j}}\right] \text {. }
$$

We always assume $A$ is nonsingular, i.e. $\Delta(f) \neq 0$. We note that $A$ is an even matrix, that is, it has integer entries and is even along the diagonal. The level of $f$ is defined to be the least positive integer $N$ such that $N A^{-1}$ is even. We put $A^{\sharp}=N A^{-1}$ and denote by $f^{\sharp}$ the integral quadratic form associated to $A^{\sharp}$. By definition, $f^{\#}$ is a primitive form, called the primitive adjoint of $f$. It should be noted that $N \mid \Delta$ is not necessarily true when $n$ is odd, since $\Delta A^{-1}$ need not be even (cf. [1], Satz 10.3). Of course, it is true that $N \mid 2 \Delta$. Moreover, since every $Z_{2}$-lattice has a one-dimensional orthogonal component when $n$ is odd ([1], p. 48), it is always true that $2 \mid \Delta$, $4 \mid N$.

Two integral quadratic forms $f, g$ in $n$ variables are equivalent if one can be obtained from the other by a linear change of variables with integer coefficients and determinant \pm 1 . Equivalent forms have the same discriminant and level. To each similitude class $\{L\}$ of lattices on $V$ we can associate an equivalence class $\left\{f_{L}\right\}$ of integral quadratic forms by setting

$$
f_{L}\left(X_{1}, \cdots, X_{n}\right)=n(L)^{-1} q\left(\sum_{i=1}^{n} X_{i} v_{i}\right),
$$

where $\left\{v_{i}\right\}$ is a $Z$-basis of $L$. It is clear that $f_{L}$ is primitive and $\Delta\left(f_{L}\right)=$ $\Delta^{\prime}(L)$. If $q$ is positive definite, then $f_{L}, f_{M}$ are equivalent $\Leftrightarrow L, M$ are similar (cf. [8], p. 33). The level of $L$ is defined to be level of $f_{L}$. Let $L^{\#}$ be the dual lattice of $L$ with respect to $B$. It is clear that

$$
f_{L^{\#}}=\left(f_{L}\right)^{\#} \text {. }
$$

From now on we assume that $n=3$. Then $V$ is similar to the pure part of some quaternion algebra $\mathfrak{A}$ over $\boldsymbol{Q}$, the quadratic form being the reduced norm $N$ (cf. [1], §5.2). Accordingly, there is no loss of generality in taking $V=\{\alpha \in \mathfrak{X} \mid T(\alpha)=0\}$, where $T$ is the reduced trace on $\mathfrak{A}$. Then 
$B(\alpha, \beta)=-T(\alpha \beta)$, the similitudes of $V$ are the mappings $\xi \mapsto c \alpha \xi \alpha^{-1}, c \in \boldsymbol{Q}^{\times}$, $\alpha \in \mathfrak{H}^{\times}$, and the orthogonal transformations are those with $c= \pm 1$. Of course, $V$ is positive definite $\Leftrightarrow \mathfrak{A}$ is definite.

To determine all maximal lattices on $V$ is suffices to determine all those having square-free norm. This corresponds to determining the 1maximal and $p$-maximal lattices on $V_{p}$ for each prime $p$. Let $d$ be the product of all finite primes $p$ such that $\mathfrak{A}_{p}$ is not split. For $p \nmid d$ we define an Eichler order to be any order $\mathfrak{S}_{p}$ of $\mathfrak{U}_{p}$ such that

$$
\mathfrak{D}_{p} \cong\left[\begin{array}{ll}
Z_{p} & Z_{p} \\
p Z_{p} & Z_{p}
\end{array}\right]
$$

An Eichler order $\mathfrak{D}_{p}$ has a unique two-sided prime ideal

$$
\mathfrak{P}=\mathfrak{D}_{p}\left[\begin{array}{ll}
0 & 1 \\
p & 0
\end{array}\right]=\left[\begin{array}{ll}
p Z_{p} & Z_{p} \\
p Z_{p} & p Z_{p}
\end{array}\right]
$$

From $\S 2$ of [9] we obtain the following classification of maximal lattices:

If $p \mid d$ : (i ) $L_{p}$ is 1-maximal $\Leftrightarrow L_{p}=\left\{\alpha_{p} \in V_{p} \mid N\left(\alpha_{p}\right) \in Z_{p}\right\}=\mathfrak{S}_{p} \cap V_{p}$, where $\mathfrak{D}_{p}$ is the unique maximal order of $\mathfrak{A}_{p}$.

(ii) $L_{p}$ is $p$-maximal $\Leftrightarrow L_{p}=\left\{\alpha_{p} \in V_{p}|p| N\left(\alpha_{p}\right)\right\}=\mathfrak{B} \cap V_{p}$, where $\mathfrak{P}$ is the unique two-sided prime ideal of $\mathfrak{D}_{p}$.

If $p \nmid d$ : (iii) $L_{p}$ is 1 -maximal $\Leftrightarrow L_{p}=\mathfrak{D}_{p} \cap V_{p}$, where $\mathfrak{D}_{p}$ is a maximal order of $\mathfrak{U}_{p}$.

(iv) $L_{p}$ is $p$-maximal $\Leftrightarrow L_{p}=\mathfrak{P} \cap V_{p}$, where $\mathfrak{P}$ is the two-sided prime ideal of an Eichler order $\mathfrak{S}_{p}$ of $\mathfrak{U}_{p}$.

The corresponding reduced discriminants $\Delta_{p}^{\prime}$ and levels $N_{p}$ are:

(i) $\Delta_{p}^{\prime}=\left(2 p^{2}\right), N_{p}= \begin{cases}(4) & \text { if } p=2 \\ (p) & \text { if } p \neq 2\end{cases}$

(ii), (iv) $\Delta_{p}^{\prime}=(2 p), N_{p}= \begin{cases}(8) & \text { if } p=2 \\ (p) & \text { if } p \neq 2\end{cases}$

(iii) $\Delta_{p}^{\prime}=(2), N_{p}=(4)$.

We observe that the lattices of type (i) are paired naturally with those of type (ii). If $L_{p}$ is of either type, then $M_{p}=\left\{\alpha_{p} \in L_{p}|p|\left(N\left(\alpha_{p}\right) / N\left(L_{p}\right)\right)\right\}$ is of the other type.

For $p \neq 2 M_{p}$ is similar to $L_{p}^{\#}$, so this pairing is ordinary duality. For $p=2$ it is not the dual pairing, since the level is changed. Indeed, 
a lattice $L_{2}$ of type (i) is similar to its dual $L_{2}^{\#}$. In accordance with this pairing, we introduce the notion of " $\delta$-transform" of a maximal lattice $L$ for any $\delta \mid d$. If $L$ is a maximal lattice on $V$, then $L^{(\delta)}=\left\{\alpha \in L|\delta| f_{L}(\alpha)\right\}$, $f_{L}^{(\delta)}=f_{L^{(\delta)}}$. Such a transform changes the type of the lattice at any prime $p \mid \delta$. These transforms are special cases of transformations introduced by Watson [13] which do not increase the class number. In the present case it is clear that two maximal lattices $L, M$ are similar $\Leftrightarrow L^{(\delta)}, M^{(\delta)}$ are similar, which means the class number is actually unchanged. The primitive adjoint of a form is another example of a Watson transformation which leaves the class number unchanged. However, the dual of maximal lattice need not be maximal. For example, the dual of a lattice of type (iv) is similar to the trace 0 part of an Eichler order, hence non-maximal.

From above we see that if $L$ is a maximal lattice on $V$, then $L$ has level $4 m$, where $m$ is a square-free multiple of $d$. The converse is not true. However, it can be shown that any lattice on $V$ with such a level is similar to one gotten from the trace 0 part of an order which is locally either maximal or Eichler by taking a finite number of $\delta$-transforms and duals. The details will appear elsewhere. In this paper we will only consider lattices which are similar to $\delta$-transforms of lattices $L$ of the following two types:

I. $L=\mathfrak{D} \cap V$ for some maximal order $\mathfrak{O}$ of $\mathfrak{A}$.

(3) II. If $d$ is odd, then $L=\left(2 d M^{\sharp}\right)^{(d)}$, where $M$ is of type I.

The lattices of type I are simply the 1-maximal lattices on $V$. If $L$ is of type II, then $L_{p}=M_{p}=\mathfrak{D}_{p} \cap V_{p}$ for $p \mid d$ but

$$
L_{2}=2 M_{2}^{\sharp} \cong\left\{\left[\begin{array}{cc}
-a & 2 b \\
2 c & a
\end{array}\right] \mid a, b, c \in Z_{2}\right\} .
$$

In particular, such a lattice has norm 1 but is not maximal. The reason for choosing $L$ in this form when $d$ is odd will become apparent in the next section. We note that the lattices of type I have discriminant $2 d^{2}$, while those of type II have discriminant $32 d^{2}$. It is clear that a lattice of reduced discriminant $\Delta$ is similar to a $\delta$-transform of a lattice of type I, type II, resp. $\Leftrightarrow 2 d|\Delta| 2 d^{2}, 32 d|\Delta| 32 d^{2}$, resp. In either case, the total number of similitude genera with such reduced discriminants is $2^{e}$, where $e=$ the number of primes dividing $d$.

If $L$ is of type I, then the maximal order $\subseteq$ such that $\subseteq \cap V=L$ is 
uniquely determined ([9], p. 585). Let $G$ be the similitude genus containing the lattices of type I. Then the number of similitude classes in $G$ is equal to $T$, "the type number" of $\mathfrak{A}$, that is, the number of isomorphism classes of maximal orders of $\mathfrak{U}([9]$, p. 586). It follows that any similitude genus obtained from $G$ by taking $\delta$-transforms and duals also has $T$ similitude classes.

\section{§2. Eichler's commutation relation for type I lattices}

Let $f=f\left(X_{1}, X_{2}, X_{3}\right)$ be a positive definite integral ternary quadratic form. Let $\Delta$ be the discriminant of $f$ and $N$ its level. For each integer $n \geq 0$ put $a(n, f)=\left\{v \in Z^{3} \mid f(v)=n\right\}$ and

$$
\theta(z)=\theta(z, f)=\sum_{n=0}^{\infty} a(n, f) e^{2 \pi i n z} .
$$

Then $\theta$ is an integral modular form of weight $3 / 2$, level $N$ and character $\chi(d)=(2 \Delta / d)([11]$, p. 456). For each prime number $p$ we have a Hecke operator $T_{3, \chi}^{N}\left(p^{2}\right)$ defined ([11], p. 450) such that if

$$
\left(\theta \mid T_{3, \chi}^{N}\left(p^{2}\right)\right)(z)=\sum_{n=0}^{\infty} b(n) e^{2 \pi i n z},
$$

then $b(n)=a\left(p^{2} n, f\right)+((-2 \Delta n) / p) a(n, f)+p a\left(n / p^{2}, f\right)$ if $p \nmid \Delta$ and

$$
b(n)=a\left(p^{2} n, f\right) \quad \text { if } p \mid \Delta .
$$

In particular, $b(n)=a(4 n, f)$ for any $n \geq 0$.

Suppose $f=f_{L}$, where $L$ is a lattice of type I. Following ideas of Eichler ([1], §21.3), we will show that $\theta \mid T_{3, x}^{N}\left(p^{2}\right)$ for $p \nmid \Delta$ is a certain explicit linear combination of $\theta$-series associated to the similitude genus of $L$. The coefficients of this linear combination come from an "Anzahlmatrix" $P\left(p^{2}\right)$, which we now describe. Let $C_{1}, \cdots, C_{T}$ be the similitude classes in $G$. Choose a representative $L_{i} \in C_{1}, i=1, \cdots, T$. Let $p$ be a prime number. Fix $i$ and consider the set of all $K \in G$ such that $K \subset L_{i}, n(K)=$ $p^{2} n\left(L_{i}\right)$, and $K \neq p L_{i}$ if $p \nmid d$. This is a finite set with a cardinality which depends on $p$ but not on $i$. We denote this cardinality by $\pi\left(p^{2}\right)$. Put $\pi_{i j}\left(p^{2}\right)=$ the number of $K$ which are similar to $L_{j}$. It is easily seen that $\pi_{i j}\left(p^{2}\right)$ depends on $(i, j)$ but not on the choice of representatives $L_{i}, L_{j}$. Then $P\left(p^{2}\right)=\left[\pi_{i j}\left(p^{2}\right)\right]$ is an "Anzahlmatrix" in the sense of Eichler ([1], p. 109), where the elementary divisors $(p, p, p)$ are not allowed for $p \nmid d$. We note that our matrices are the transposes of Eichler's. For any $i$ we have 


$$
\sum_{j=1}^{T} \pi_{i j}\left(p^{2}\right)=\pi\left(p^{2}\right)
$$

For any lattice $L$ on $V$ denote $a\left(n, f_{L}\right)$ by $a(n, L)$ and $\theta\left(z, f_{L}\right)$ by $\theta(z, L)$. Set

$$
\theta_{G}(z)=\left[\begin{array}{c}
\theta\left(z, L_{1}\right) \\
\vdots \\
\theta\left(z, L_{T}\right)
\end{array}\right] .
$$

Proposition 1. (Eichler's commutation relation.) Let $G$ be the similitude genus containing the lattices of type $\mathrm{I}$. Let $\Delta$ be the reduced discriminant of $G, N$ the level of $G, \chi$ the character $(\bmod N)$ defined by $\chi(d)=$ $(2 \Delta / d)$. Then

$$
\theta_{G} \mid T_{3, \alpha}^{N}\left(p^{2}\right)=P\left(p^{2}\right) \theta_{G}
$$

for any prime $p \nmid \Delta$.

Although Eichler's book [1] does not contain the definition of Hecke operators for half integral weight, the above "commutation relation" is, in principle, contained as a special case of his formula $18.20 \mathrm{C}$, p. 123 . For the sake of exposition and the convenience of the reader, we present a self-contained proof here. Since $2 \Delta=4 d^{2}$, a square, we must show

$$
\sum_{j=1}^{T} \pi_{i j}\left(p^{2}\right) a\left(n, L_{j}\right)=a\left(p^{2} n, L_{i}\right)+\left(\frac{-n}{p}\right) a\left(n, L_{i}\right)+p a\left(n / p^{2}, L_{i}\right)
$$

for any $n \geq 0$.

We may assume the $L_{i}$ are of type I, so that $L_{i}=\mathfrak{S}_{i} \cap V, i=1, \cdots, T$, where $\mathfrak{D}_{1}, \cdots, \mathfrak{D}_{T}$ form a complete set of representatives for the isomorphism classes of maximal orders of $\mathfrak{Y}$. Let $(5)$ be the set of all rational multiples of maximal orders of $\mathfrak{X}$. Then we have a one-to-one correspondence between the elements $\AA$ of $(s)$ and $K$ of $G$ given by $\Re \mapsto \Re \cap V=K$. Suppose $L$ is of type I, $L=\mathfrak{O} \cap V, \mathfrak{O}$ a maximal order of $\mathfrak{A}$. Then the problem of determining all $K \in G$ such that $K \subset L, n(K)=p^{2} n(L)=p^{2}, K \neq$ $p L$ is the same as determining all $\Re \in \mathbb{S}$ such that $\Re \subset S, n(\Re)=p^{2}, \Re \neq$ $p \lesssim$. For any such $\Re$ we must have

$$
\begin{array}{ll}
\Re_{q}=\mathfrak{D}_{q} & \text { for } q \neq p \\
\mathfrak{\Re}_{p}=p \beta_{p}^{-1} \mathfrak{D}_{p} \beta_{p} & \text { for some } \beta_{p} \in \mathfrak{A}_{p}^{\times} .
\end{array}
$$

We may assume $\mathfrak{A}_{p}=M\left(2, \boldsymbol{Q}_{p}\right), \mathfrak{O}_{p}=M\left(2, Z_{p}\right)$. By direct computation, 
$p \beta_{p}^{-1} M\left(2, Z_{p}\right) \beta_{p} \subset M\left(2, Z_{p}\right)$ implies $\beta_{p}=c \alpha_{p}$, where $c \in \boldsymbol{Q}_{p}^{\times}, \alpha_{p} \in M\left(2, Z_{p}\right)$ and

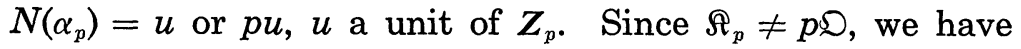

$$
\Re_{p}=p \alpha_{p}^{-1} \mathfrak{D}_{p} \alpha_{p},
$$

where $\alpha_{p} \in \mathfrak{D}_{p}$ and $N\left(\alpha_{p}\right)=p u, u$ a $p$-adic unit. Multiplying $\alpha_{p}$ on the left by a suitable unit of $\mathfrak{D}_{p}$, we may assume

$$
\alpha_{p}=\left[\begin{array}{ll}
p & 0 \\
0 & 1
\end{array}\right] \text { or } \alpha_{p}=\left[\begin{array}{ll}
1 & c \\
0 & p
\end{array}\right], c \in Z, 0 \leq c<p .
$$

Each of these $\alpha_{p}$ gives a distinct sublattice $\Re$ of $\mathscr{O}$ of the required type. In particular, we have

$$
\pi\left(p^{2}\right)=p+1 \text {. }
$$

Then (6) combined with (11) verifies (8) for $n=0$.

Suppose $L$ is of type $I, L=\subseteq \cap V$, $\subseteq$ a maximal order. For each integer $n \geq 1$ let $A(n, L)=\{\nu \in L \mid N(\nu)=n\}=\left\{\nu \in \mathfrak{D} \mid \nu^{2}=-n\right\}$, so that

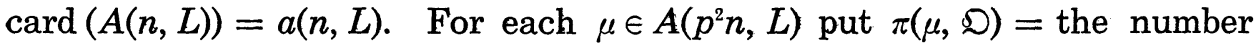
of $\AA_{p}$ such that $\mu \in \Re_{p}$. We say that two vectors $\lambda, \lambda^{\prime} \in L_{p}$ are associates, and write $\lambda \sim \lambda^{\prime}$, if $\lambda^{\prime}=\sigma(\lambda)$ for some orthogonal automorphism $\sigma$ of $L_{p}$. Then $\pi(\mu, \mathcal{D})=$ the number of $\Omega_{p}$ containing $\mu^{\prime}$ for any associate $\mu^{\prime}$ of $\mu$. For an integer $k \geq 0$ we write $p^{k} \mid \mu$ if $p^{-k} \mu \in \mathcal{D}_{p}$; we write $p^{k} \| \mu$ if $p^{k} \mid \mu$ but $p^{k+1} \chi \mu$. In particular, $1 \| \mu \Leftrightarrow \mu$ is a primitive vector of $\mathfrak{D}_{p}$ (or $L_{p}$ ). Since $p \nmid \Delta\left(L_{p}\right)$, any two primitive vectors in $L_{p}$ of the same length are associates ([1], p. 60). It follows that $p^{k} \| \mu \Leftrightarrow \mu \sim \mu^{\prime}$, where

$$
\mu^{\prime}=p^{k}\left[\begin{array}{rc}
0 & m \\
-1 & 0
\end{array}\right]
$$

with $m=n p^{2(1-k)}$.

Lemma. Let $\mu \in A\left(p^{2} n, L\right)$. Then

$$
\pi(\mu, D)=\left\{\begin{array}{cl}
1 & \text { if } \mu \text { is primitive, } \\
1+\left(\frac{-n}{p}\right) & \text { if } p \| \mu, \\
p+1 & \text { if } p^{2} \mid \mu .
\end{array}\right.
$$

Proof. Suppose $p^{k} \| \mu$. Taking $\Re_{p}$ as in (9) and $\mu^{\prime}$ as in (12), we see

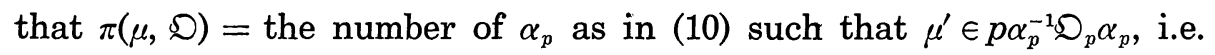

$$
p^{k-1} \alpha_{p}\left[\begin{array}{rc}
0 & m \\
-1 & 0
\end{array}\right] \alpha_{p}^{-1} \in M\left(2, Z_{p}\right)
$$


If $\mu$ is primitive, then $k=0$ and (14) holds only for

$$
\alpha_{p}=\left[\begin{array}{ll}
1 & 0 \\
0 & p
\end{array}\right]
$$

If $k>1$, then $p^{k-1} \alpha_{p}^{-1} \in M\left(2, Z_{p}\right)$ for every $\alpha_{p}$, hence (14) holds for every $\alpha_{p}$, and $\pi(\mu, \mathcal{D})=\pi\left(p^{2}\right)=p+1$. Now suppose $k=1$, so that $m=n$. Then (14) is valid for $\alpha_{p}=\left[\begin{array}{ll}p & 0 \\ 0 & 1\end{array}\right]$. Taking $\alpha_{p}=\left[\begin{array}{ll}1 & c \\ 0 & p\end{array}\right], 0 \leq c<p$, we get

$$
\alpha_{p}\left[\begin{array}{rr}
0 & n \\
-1 & 0
\end{array}\right] \alpha_{p}^{-1}=\left[\begin{array}{cc}
-c & \left(c^{2}+n\right) p^{-1} \\
-p & c
\end{array}\right],
$$

which is in $M\left(2, Z_{p}\right) \Leftrightarrow c^{2}+n \equiv 0(\bmod p)$. Thus

$$
\pi(\mu, D)= \begin{cases}0 & \text { if }\left(\frac{-n}{p}\right)=-1 \\ 1 & \text { if } p \mid n \\ 2 & \text { if }\left(\frac{-n}{p}\right)=1 .\end{cases}
$$

Proof of Proposition 1. Let us write $A_{j}=A\left(n, L_{j}\right), B_{i}=A\left(p^{2} n, L_{i}\right)$. Note that if $K \in G$ with $n(K)=p^{2}$ is similar to $L_{j}$ (written $K \sim L_{j}$ ), then $a\left(p^{2} n, K\right)=a\left(n, L_{j}\right)$. On the one hand, we have

$$
\begin{aligned}
\sum_{\mu \in B_{i}} \pi\left(\mu, \bigcirc_{i}\right) & =\sum_{\Re} \sum_{\mu \in \Re} 1=\sum_{K} \sum_{\mu \in K} 1=\sum_{j=1}^{T} \sum_{K \sim L_{j}} \sum_{\mu \in K} 1 \\
& =\sum_{j=1}^{T} \sum_{K \sim L_{j}} a\left(n, L_{j}\right)=\sum_{j=1}^{T} a\left(n, L_{j}\right) \sum_{K \sim L_{j}} 1 \\
& =\sum_{j=1}^{T} a\left(n, L_{j}\right) \pi_{i j}\left(p^{2}\right) .
\end{aligned}
$$

On the other hand,

$$
\begin{aligned}
\sum_{\mu \in B_{i}} \pi\left(\mu, \mathfrak{D}_{i}\right) & =\sum_{1 \| \mu} \pi\left(\mu, \mathfrak{D}_{i}\right)+\sum_{p \| \mu} \pi\left(\mu, \mathfrak{D}_{i}\right)+\sum_{p^{2} \mid \mu} \pi\left(\mu, \mathfrak{D}_{i}\right) \\
& =\sum_{1 \| \mu} 1+\sum_{p \| \mu}\left(1+\left(\frac{-n}{p}\right)\right)+\sum_{p^{2} \mid \mu}(1+p),
\end{aligned}
$$

by the Lemma. Then (15) equals

$$
\begin{array}{rlr}
\sum_{\mu \in B_{i}} 1+ & \left(\frac{-n}{p}\right) \sum_{p \| \mu} 1+p \sum_{p^{2} \uparrow \mu} 1 & \\
= & a\left(p^{2} n, L_{i}\right)+p a\left(n / p^{2}, L_{i}\right) & \text { if } p \mid n, \text { and } \\
& a\left(p^{2} n, L_{i}\right)+\left(\frac{-n}{p}\right) \sum_{p \| \mu} 1 & \text { if } p \nmid n,
\end{array}
$$


in which case $p \mid \mu \Leftrightarrow p \| \mu$, so that (16) equals

$$
a\left(p^{2} n, L_{i}\right)+\left(\frac{-n}{p}\right) a\left(n, L_{i}\right),
$$

completing the proof.

Proposition 2. Let the assumptions be as in Proposition 1. Then Eichler's commutation relation (7) is valid for $p \mid d$.

Proof. We must show (cf. (5))

$$
\sum_{j=1}^{T} \pi_{i j}\left(p^{2}\right) a\left(n, L_{j}\right)=a\left(p^{2} n, L_{i}\right)
$$

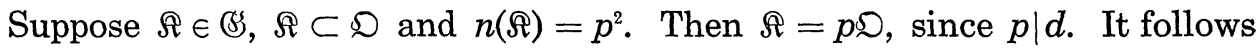
that $\pi_{i j}\left(p^{2}\right)=\delta_{i j}$, the Kronecker delta. On the other hand, for $p \mid d$ we have

$$
p^{2}|N(\mu) \Leftrightarrow p| \mu \quad \text { for } \mu \in \mathfrak{S}_{i},
$$

which implies $a\left(p^{2} n, L_{i}\right)=a\left(n, L_{i}\right)$, proving (17).

\section{§3. Eichler's commutation relation for type II lattices}

The remaining case for type I lattices where we don't have a commutation relation is $p \mid \Delta, p \nmid d$. This occurs only if $d$ is odd and $p=2$. As we shall see, (17) is not valid for $p=2$ except for special values of $n$. For general $n$ the right hand side of (17) involves representation numbers of the lattice of type II associated to $L_{i}$. In this section we derive commutation relations for type II lattices and, as a result, obtain the missing one for type I lattices.

Let $L_{1}, \cdots, L_{T}$ be lattices of type I representing the similitude classes in $G$, and $\mathfrak{D}_{1}, \cdots, \mathfrak{D}_{T}$ the corresponding maximal orders of $\mathfrak{Y}$. For each lattice $L$ of type I put

$$
L^{*}=\left(2 d L^{\sharp}\right)^{(d)},
$$

a lattice of type II (cf. (3)). Then $L_{1}^{*}, \cdots, L_{T}^{*}$ form a complete set of representatives for the similitude classes in $G^{*}$, the similitude genus containing the type II lattices. For each prime $p$ let $P^{*}\left(p^{2}\right)=\left[\pi_{i j}^{*}\left(p^{2}\right)\right]$ denote the Anzahlmatrix corresponding to norm $p^{2}$ and the system $L_{1}^{*}, \cdots, L_{T}^{*}$ (in $\S 5$ we'll see that $\left.P^{*}\left(p^{2}\right)=P\left(p^{2}\right)\right)$. Let $\theta_{G^{*}}$ be the column of $\theta$-series corresponding to $L_{1}^{*}, \cdots, L_{T}^{*}$, resp. These $\theta$-series have the same level and 
character as those from $G$.

Proposition 3. Suppose $d$ is odd. Let $N=4 d, \chi$ the character $\bmod N$ defined by $\chi(m)=\left(4 d^{2} / m\right)$. Let $G^{*}$ be the similitude genus containing the lattices of type II. Then

$$
\theta_{G^{*}} \mid T_{3, \mathrm{x}}^{N}\left(p^{2}\right)=P^{*}\left(p^{2}\right) \theta_{G^{*}} \quad \text { for } p \neq 2
$$

and

$$
\theta_{G^{*}} \mid T_{3, x}^{N}(4)=\theta_{G} \text {. }
$$

Proof. If $L$ is of type I, then $L_{p}^{*}=L_{p}$ for all $p \neq 2$. The arguments used to prove Propositions 1 and 2 carry over verbatim to prove (18). It is evident from (4) that $a\left(4 n, L^{*}\right)=a(n, L)$, which proves (19).

The identity (19) is not the commutation relation we seek, as it does not involve $P^{*}(4)$. Before stating it we need some preparatory discussion. We may assume $\mathfrak{U}_{2}=M\left(2, Q_{2}\right), \mathfrak{D}_{2}=M\left(2, Z_{2}\right)$. Then

$$
\begin{gathered}
L_{2}=\left\{\left[\begin{array}{rr}
-a & b \\
c & a
\end{array}\right] \mid a, b, c \in Z_{2}\right\}, \\
L_{2}^{*}=2 L_{2}^{\#}=\left\{\left[\begin{array}{cc}
-a & 2 b \\
2 c & a
\end{array}\right] \mid a, b, c \in Z_{2}\right\} .
\end{gathered}
$$

By duality, the orthogonal automorphisms of $L_{2}^{*}$ are the same as those of $L_{2}: \xi \mapsto \pm \varepsilon^{-1} \xi \varepsilon$, where $\varepsilon$ is a unit of $\mathfrak{D}_{2}$. Suppose $K \in G^{*}, K \subset L^{*}, n(K)$ $=4, K \neq 2 L^{*}$. Then $K_{p}=L_{p}^{*}$ for $p \neq 2$ and

$$
K_{2}=2 \beta_{2}^{-1} L_{2}^{*} \beta_{2}
$$

for some $\beta_{2} \in G L(2, \boldsymbol{Q})$. However, $2 \beta_{2}^{-1} L_{2}^{*} \beta_{2} \subset L_{2}^{*} \Leftrightarrow 2 \beta_{2}^{-1} L_{2}^{\#} \beta_{2} \subset L_{2}^{\#} \Leftrightarrow 2^{-1} \beta_{2}^{-1} L_{2} \beta_{2}$ $\supset L_{2} \Leftrightarrow L_{2} \supset 2 \beta_{2} L_{2} \beta_{2}^{-1} \Leftrightarrow \mathfrak{D}_{2} \supset 2 \beta_{2} \bigcirc_{2} \beta_{2}^{-1}$. As before, we may replace $\beta_{2}$ by $\alpha_{2}$ $\in \mathfrak{D}_{2}$ with $N\left(\alpha_{2}\right)=2 u, u$ a 2-adic unit. Multiplying $\alpha_{2}$ on the left by a suitable unit of $\mathfrak{S}_{2}$, we may assume $\alpha_{2}$ is as in (10) with $p=2$.

Lemma 1. Suppose $\lambda, \lambda^{\prime}$ are two primitive vectors of $L_{2}^{*}$ such that $N(\lambda)$ $=N\left(\lambda^{\prime}\right)$ and $\lambda \equiv \lambda^{\prime}\left(\bmod 2 L_{2}\right)$. Then $\lambda \sim \lambda^{\prime}$.

Proof. We apply Kneser's criterion ([5], § 2) to the situation $E=L_{2}^{*}$, $a=4, F=(\lambda), G=\left(\lambda^{\prime}\right)$. Then $E^{a}=E_{a}=2 L_{2}$. Kneser's condition (9) follows from the primitivity of $\lambda, \lambda^{\prime}$.

For an element $\mu \in A\left(4 n, L^{*}\right)$ put $\pi\left(\mu, L^{*}\right)=$ the number of $K$ as above such that $\mu \in K_{2}$. Then $\pi\left(\mu, L^{*}\right)$ is the number of $\alpha_{2}$ as in (10) satisfying 


$$
2^{-1} \alpha_{2} \mu^{\prime} \alpha_{2}^{-1} \in L_{2}^{*}
$$

for any fixed associate $\mu^{\prime}$ of $\mu$. We note that if $n$ is represented by $L^{*}$, then $-n$ must be a square $(\bmod 4)$, so that $n \equiv 0,3(\bmod 4)$. For such a value of $n$ let $(-n / 2)$ denote the usual Kronecker symbol.

Lemma 2. Let $\mu \in A\left(4 n, L^{*}\right)$, where $n \equiv 0,3(\bmod 4)$. Then

$$
\pi\left(\mu, L^{*}\right)=\left\{\begin{array}{cl}
1 & \text { if } \mu \text { is primitive, } \\
1+\left(\frac{-n}{2}\right) & \text { if } 2 \| \mu, \\
3 & \text { if } 4 \| \mu .
\end{array}\right.
$$

Proof. Suppose $\mu=\left[\begin{array}{cr}-x & 2 y \\ 2 z & x\end{array}\right]$. Then $-x^{2}-4 y z=4 n$ implies that $2 \mid x$. Moreover, $\mu$ is primitive $\Leftrightarrow(y, z)=1$. Suppose $\mu$ is primitive. Applying Kneser's criterion, we may take

$$
\mu^{\prime}=\left[\begin{array}{rc}
0 & 2 n \\
-2 & 0
\end{array}\right]
$$

Checking condition (21), we find that $\pi\left(\mu, L^{*}\right)=1$.

Now suppose $2^{k} \| \mu, k \geq 1$. If $k>1$, then all of the $\alpha_{2}$ satisfy (21), so that $\pi\left(\mu, L^{*}\right)=3$ in this case.

Finally suppose $k=1$. Then $\mu=2\left[\begin{array}{rr}-x & 2 y \\ 2 z & x\end{array}\right]$, where $(x, y, z)=1$ and $-x^{2}-4 y z=n$. There are two cases:

(1) If $2 \mid x$, then $n \equiv 0(\bmod 4)$ and we may take

$$
\mu^{\prime}=\left[\begin{array}{rc}
0 & n / 2 \\
-2 & 0
\end{array}\right]
$$

Condition (21) is satisfied only once for each possible $n$, so $\pi\left(\mu, L^{*}\right)=1$.

(2) If $x$ is odd, then $n \equiv-1(\bmod 4)$ and we may take

$$
\mu^{\prime}=\left[\begin{array}{cc}
-1 & (n+1) / 2 \\
-2 & 1
\end{array}\right]
$$

We find that $\pi\left(\mu, L^{*}\right)=0$ if $-n \equiv 5(\bmod 8)$ and $\pi\left(\mu, L^{*}\right)=2$ if $-n \equiv 1$ (mod 8). This completes the proof of the Lemma.

Proposition 4. Suppose $d$ is odd. Let $L_{1}^{*}, \cdots, L_{T}^{*}$ represent the isometry classes of type II lattices. Let $n \equiv 0,3(\bmod 4)$. Then

$$
\sum_{j=1}^{T} \pi_{i j}^{*}(4) a\left(n, L_{j}^{*}\right)=a\left(4 n, L_{i}^{*}\right)+\left(\frac{-n}{2}\right) a\left(n, L_{i}^{*}\right)+2 a\left(n / 4, L_{i}^{*}\right) .
$$


Proof. Using the same counting argument as for Proposition 1, we see that (23) is a direct consequence of Lemma 2.

Corollary. $P^{*}(4) \theta_{G}=\theta_{G} \mid T_{3, x}^{N}(4)+2 \theta_{G^{*}}$.

Proof. We have for any $n$ :

$$
\begin{aligned}
\sum_{j=1}^{T} \pi_{i j}^{*}(4) a\left(n, L_{j}\right) & =\sum_{j=1}^{T} \pi_{i j}^{*}(4) a\left(4 n, L_{j}^{*}\right)=a\left(16 n, L_{i}^{*}\right)+2 a\left(n, L_{i}^{*}\right) \\
& =a\left(4 n, L_{i}\right)+2 a\left(n, L_{i}^{*}\right) .
\end{aligned}
$$

In particular, the Corollary shows that the space of theta series associated to lattices of type $I$ is not invariant under the Hecke operator $T_{3, \chi}^{N}(4)$ when $d$ is odd. As a special case of (24) we have

$$
\sum_{j=1}^{T} \pi_{i j}^{*}(4) a\left(n, L_{j}\right)=a\left(4 n, L_{i}\right)+2 a\left(n / 4, L_{i}\right) \quad \text { if } 4 \mid n
$$

\section{§4. Brandt matrices}

In this section we show that the Anzahlmatrices defined in $\S 2$ are nothing but "reduced" Brandt matrices associated to the quaternion algebra $\mathfrak{A}$. First we recall the definition of a Brandt matrix (cf. [3], p. 138). Let $\mathfrak{O}$ be a maximal order of $\mathfrak{A}$. Two left $\mathfrak{D}$-ideals $\mathfrak{L}, \mathfrak{L}^{\prime}$ are equivalent if $\mathfrak{L}^{\prime}=\mathfrak{L} \alpha$ for some $\alpha \in \mathfrak{U}^{\times}$. An analogous notion of equivalence can be defined for right $\mathfrak{D}$-ideals. It is well known that the number of equivalence classes of left $\mathfrak{O}$-ideals is the same as the number of equivalence classes of right $\mathfrak{O}$-ideals, and that this number is independent of the choice of maximal order $\mathfrak{D}$. Fix a maximal order $\mathfrak{D}$ and let $\mathfrak{D}=\mathfrak{\Omega}_{1}, \cdots, \mathfrak{\Omega}_{H}$ be a complete set of representatives for the left ideal classes of $\mathfrak{D}$. Let $\mathfrak{O}=$ $\mathfrak{D}_{1}, \cdots, \mathfrak{O}_{H}$ be the right orders of $\mathfrak{\Omega}_{1}, \cdots, \mathfrak{R}_{H}$, resp. Since any maximal order is isomorphic to one of the $\mathfrak{S}_{i}$, there is no loss of generality in assuming that $\mathfrak{D}_{1}, \cdots, \mathfrak{D}_{T}$ give a complete set of representatives for the isomorphism classes of maximal orders of $\mathfrak{A}$. We note, in particular, that $T \leq H$.

We put $\mathfrak{L}_{k \ell}=\mathfrak{L}_{k}^{-1} \mathfrak{Q}_{\ell}, 1 \leq k, \ell \leq H$, a lattice on $\mathfrak{A}$ with left order $\mathfrak{O}_{k}$ and right order $\mathfrak{D}_{\ell}$. If we fix $k$, then $\mathfrak{\Omega}_{k \ell}, \ell=1, \cdots, H$, give a complete set of representatives for the left ideal classes of $\mathfrak{D}_{k}$. We recall that any proper similitude of $\mathfrak{A}$ is of the form $\xi \mapsto \alpha \xi \beta, \alpha, \beta \in \mathfrak{A}^{\times}$([8], p. 4). It follows that any lattice on $\mathfrak{A}$ of reduced discriminant $d^{2}$ is properly similar to one of the $\mathfrak{\Omega}_{k \ell}$. Let $n$ be a positive integer. The Brandt matrix $B(n)$ 
corresponding to $n$ is defined by;

$$
B(n)=\left[b_{k \ell}(n)\right] \text {, }
$$

where $b_{k \ell}(n)=$ the number of integral left $\mathfrak{N}_{k}$-ideals of norm $n$ which are equivalent to $\mathfrak{R}_{k \ell}$. Since $\mathfrak{\Omega}_{k \ell}^{-1}=\mathfrak{\Omega}_{\ell k}$, it is apparent that $b_{k \ell}(n)=$ the number of left $\mathfrak{D}_{k}$-ideals of the form $\mathfrak{I}_{k \ell} \alpha$, where $\alpha \in \mathfrak{\Omega}_{\ell k}$ and $N\left(\mathfrak{\Omega}_{k \ell} \alpha\right)=n$, i.e. $N(\alpha) / N\left(\mathfrak{Q}_{\ell k}\right)$ $=n$. Let $f_{\ell k}$ be the integral quadratic form associated to $\mathfrak{\Omega}_{\ell k}$ as in (1). Let $w_{\ell}=a\left(1, f_{\ell \ell}\right)=$ order of the unit group of $\mathfrak{D}_{\ell}$. Then

$$
b_{k \ell}(n)=\frac{a\left(n, f_{\ell k}\right)}{w_{\ell}} \quad \text { for } n \geq 1
$$

and if we set $b_{k \ell}(0)=1 / w_{\ell}$, we obtain

$$
\sum_{n=0}^{\infty} b_{k \ell}(n) e^{2 \pi i n z}=\frac{1}{w_{\ell}} \theta\left(z, f_{\ell k}\right),
$$

an integral modular form of weight 2 , level $d$ and trivial character.

As before, let $L_{i}=\mathfrak{O}_{i} \cap V, i=1, \cdots, T$. Let $p$ be a prime not dividing d. Fix $i, j \leq T$. Any similitude of norm $p^{2}$ on $V$ is of the form $\xi \mapsto p \alpha^{-1} \xi \alpha$, $\alpha \in \mathfrak{U}^{\times}$. It follows that $\pi_{i j}\left(p^{2}\right)=$ the number of sublattices $K \subset L_{i}$ of the form $K=p \alpha^{-1} L_{j} \alpha, K \neq p L_{i}$. By the one-to-one correspondence between $G$ and $\mathbb{S}$, the latter equals the number of sublattices $\Re \subset \mathfrak{O}_{i}$ of the form $p \alpha^{-1} \mathfrak{D}_{j} \alpha \neq p \mathfrak{D}_{i}$, i.e. $\alpha^{-1} \mathfrak{D}_{j} \alpha \neq \mathfrak{N}_{i}$ (this is automatically satisfied if $i \neq j$ ). Our basic problem is to determine all $\alpha \in \mathfrak{H}^{\times}$such that

$$
p \alpha^{-1} \mathfrak{D}_{j} \alpha \subset \mathfrak{D}_{i}
$$

We first solve this problem in the idele group $J_{\mathfrak{R}}$ of $\mathfrak{A}$, that is, we find all $\tilde{\alpha}=\left(\alpha_{q}\right) \in J_{\mathscr{Q}}$ such that

$$
p \tilde{\alpha}^{-1} \mathfrak{D}_{j} \tilde{\alpha} \subset \mathfrak{D}_{i},
$$

where $p \tilde{\alpha}^{-1} \mathfrak{D}_{j} \tilde{\alpha}$ is the lattice on $\mathfrak{U}$ whose $q$-th localization is $p \alpha_{q}^{-1}\left(\mathfrak{D}_{j}\right)_{q} \alpha_{q}$.

For any maximal order $\mathfrak{O}$ put $\mathfrak{N}(\tilde{\mathfrak{D}})=\left\{\tilde{\gamma} \in J_{\mathfrak{l l}} \mid \tilde{\gamma}^{-1} \mathfrak{⿴} \tilde{\gamma}=\mathfrak{D}\right\}, \mathfrak{N}(\mathfrak{D})=\mathfrak{N}(\tilde{\mathfrak{D}})$ $\cap \mathfrak{U}^{\times}$. It is well known (cf. [7], p. 299) that $\tilde{\gamma} \in \mathfrak{N}(\tilde{D}) \Leftrightarrow \tilde{\gamma}=r \tilde{\varepsilon} \tilde{\delta}$, where $r \in$

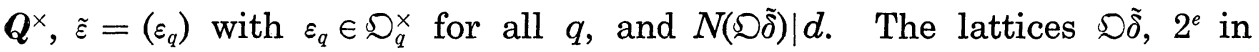
number, give a complete set of representatives for the group of two-sided ideals of $\mathfrak{D}$ mod the rational principal ones.

Since $\mathfrak{D}_{j}, \mathfrak{D}_{i}$ are locally isomorphic, $\exists \tilde{\alpha}_{0} \in J_{\mathscr{V}}$ such that $\tilde{\alpha}_{0}^{-1} \mathfrak{D}_{j} \tilde{\alpha}_{0}=\mathfrak{S}_{i}$. Then 


$$
p \tilde{\alpha}^{-1} \mathfrak{D}_{j} \tilde{\alpha} \subset \mathfrak{D}_{i} \Rightarrow p \tilde{\alpha}^{-1} \mathfrak{D}_{j} \tilde{\alpha} \subset \tilde{\alpha}_{0}^{-1} \mathfrak{D}_{j} \tilde{\alpha}_{0} \Rightarrow p\left(\tilde{\alpha} \tilde{\alpha}_{0}^{-1}\right)^{-1} \mathfrak{D}_{j} \tilde{\alpha} \tilde{\alpha}_{0}^{-1} \subset \mathfrak{D}_{j} \Rightarrow \tilde{\alpha} \tilde{\alpha}_{0}^{-1}=\tilde{\gamma} \tilde{\beta},
$$

where $\tilde{\gamma} \in \mathfrak{N}\left(\tilde{\mathfrak{D}}_{j}\right)$ and $N\left(\mathfrak{D}_{j} \tilde{\beta}\right)=p$. Hence

$$
\tilde{\alpha}=r \tilde{\varepsilon} \tilde{\delta} \tilde{\beta} \tilde{\alpha}_{0},
$$

where $r, \tilde{\varepsilon}, \tilde{\delta}$ are as above for $\mathfrak{D}=\mathfrak{D}_{j}$. Put $\mathfrak{\Im}_{0}=\mathfrak{O}_{j} \tilde{\alpha}_{0} . \mathfrak{D}=\mathfrak{O}_{j} \tilde{\delta}$. Then $\mathfrak{S}_{0}$ is a left $\mathfrak{D}_{j}$-ideal with right order $\mathfrak{D}_{i}$, and $\mathfrak{D}$ is a two-sided ideal of $\mathfrak{D}_{j}$ with $N(\mathfrak{D}) \mid d$. Suppose $\alpha \in \mathfrak{U}^{\times}$satisfies (28). Then (30) implies that

$$
\alpha \in r \mathfrak{S} \mathfrak{S}_{0}
$$

for some $r \in \boldsymbol{Q}^{\times}$and $\mathfrak{D}$, such that

$$
N(\alpha)=p N\left(r \cong \Im_{0}\right) \text {. }
$$

Multiplying $\alpha$ on the left by an element of $\mathfrak{N}(\mathfrak{D})$ leaves $p \alpha^{-1} \mathfrak{N}_{j} \alpha$ unchanged. Hence we may assume $r=1$ and $\{D$ \} is a complete set of representatives for the two-sided ideals of $\mathfrak{S}_{j}$ mod the principal ones, a group of order $2^{e-f_{j}}$ for some $0 \leq f_{j} \leq e$. If we fix $\mathfrak{S}_{0}$ and let $\mathfrak{D}$ vary, then $\mathfrak{S}=\mathfrak{D} \mathfrak{S}_{0}$ will range over a complete set of representatives for the right $\mathfrak{S}_{i}$-ideal classes with left order isomorphic to $\mathfrak{D}_{j}$. This shows, in particular, that

$$
\sum_{j=1}^{T} 2^{e-f_{j}}=H
$$

LEMMA. Let $\{\mathfrak{S}\}$ be a complete set of representatives for the right $\mathfrak{D}_{i}$ ideals with left order isomorphic to $\mathfrak{D}_{j}$. Let $p$ be a prime, $p \nmid d$. Then

$$
\pi_{i j}\left(p^{2}\right)=\sum_{\Im} \frac{a\left(p, f_{\Im}\right)}{w_{j}}
$$

Proof. Multiplying each $\mathfrak{S}$ on the left by a suitable element of $\mathfrak{U}^{\times}$, we may assume $\{\mathfrak{J}\}$ is given as in the preceding discussion. Then for each $\Re$ we have an ideal $\mathfrak{S}$ and an element $\alpha \in \mathfrak{S}$ such that $N(\alpha)=p N(\mathfrak{S})$ and $p \alpha^{-1} \mathfrak{D}_{j} \alpha=\Re$. Suppose $\alpha_{1} \in \widetilde{\Im}_{1}, \alpha_{2} \in \widetilde{\mathfrak{V}}_{2}, N\left(\alpha_{1}\right)=p N\left(\widetilde{\mathfrak{S}}_{1}\right), N\left(\alpha_{2}\right)=p N\left(\widetilde{\Im}_{2}\right)$, and

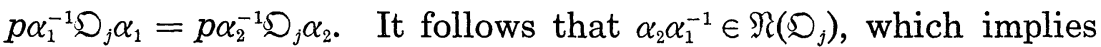

$$
N\left(\widetilde{\mathfrak{J}}_{2} \widetilde{\mathfrak{J}}_{1}^{-1}\right)=N\left(\alpha_{2} \alpha_{1}^{-1}\right) \in N\left(\mathfrak{N}\left(\mathfrak{D}_{j}\right)\right) \text {. }
$$

Since the two-sided ideals of $\mathfrak{D}_{j}$ are uniquely determined by their norms, (35) $\Rightarrow \widetilde{S}_{2}, \mathfrak{S}_{1}$ are equivalent $\Rightarrow \widetilde{S}_{2}=\mathfrak{S}_{1}$. Then $\alpha_{2} \alpha_{1}^{-1} \in \mathfrak{S}_{2} \mathfrak{\Im}_{1}^{-1}=\mathfrak{O}_{j}$ and $N\left(\alpha_{2} \alpha_{1}^{-1}\right)$ $=1$, which means $\alpha_{2}=\varepsilon \alpha_{1}$ for some $\varepsilon \in \mathfrak{D}_{j}^{\times}$. This completes the proof.

Proposition 5. Let $p$ be a prime. Then 


$$
\pi_{i j}\left(p^{2}\right)=\sum_{D_{\ell} \cong D_{j}} b_{i \ell}(p) .
$$

Proof. First supppose $p \nmid d$. As $\ell$ varies over all indices such that $\mathfrak{D}_{\ell} \cong \mathfrak{D}_{j}, \mathfrak{R}_{\ell i}$ varies over a complete set of representatives for the right $\mathfrak{D}_{i}$ -ideals with left order isomorphic to $\mathfrak{D}_{j}$. Therefore, by the Lemma and (27),

$$
\pi_{i j}\left(p^{2}\right)=\sum_{D_{\ell} \equiv D_{j}} \frac{a\left(p, f_{\ell i}\right)}{w_{\ell}}=\sum_{\mathcal{O}_{\ell} \equiv_{j}} b_{i \ell}(p) .
$$

Now suppose $p \mid d$. Then, as remarked earlier, $\pi_{i j}\left(p^{2}\right)=\delta_{i j}$. On the other hand, since an integral left $\mathfrak{D}_{i}$-ideal of norm $p$ is uniquely determined and two-sided, $b_{i \ell}(p)=0$ unless $\mathfrak{D}_{\ell} \cong \mathfrak{O}_{i}$ and, in that case, $b_{i \ell}(p)=1$ for a unique $\ell$.

Applying Proposition 5, we see that the Anzahlmatrix $P\left(p^{2}\right)$ may be obtained from the Brandt matrix $B(p)$ as follows: First, for each $j \leq T$, replace the $j$-th column of $B(p)$ by the sum of all columns with index $\ell$ such that $\mathfrak{O}_{\ell} \cong \mathfrak{O}_{j}$. Then $P\left(p^{2}\right)$ is the $T \times T$ submatrix of the latter matrix consisting of all entries with row and column index $\leq T$. In this sense, we may regard $P\left(p^{2}\right)$ as a "reduced" Brandt matrix. Symbolically, we write

$$
P\left(p^{2}\right)=\overline{B(p)} \text {. }
$$

Of course, this reduction procedure can be applied to any Brandt matrix $B(n)$ to obtain a matrix $\overline{B(n)}=\left[\pi_{i j}\left(n^{2}\right)\right]$, where

$$
\pi_{i j}\left(n^{2}\right)=\sum_{\emptyset_{\ell} \cong D_{j}} b_{i \ell}(n) .
$$

Extending definition (38) to $i>T$, we see that

$$
\pi_{k j}\left(n^{2}\right)=\pi_{\ell j}\left(n^{2}\right) \quad \text { if } \mathfrak{D}_{k} \cong \mathfrak{D}_{\ell} .
$$

LEMmA. $\overline{B(m)} \overline{B(n)}=\overline{B(m) B(n)}$.

Proof. Let $B(m) B(n)=\left(c_{k \ell}\right), \overline{\left(c_{k \ell}\right)}=\left(\rho_{i j}\right) . \quad$ Let $\ell \sim j$ signify $\mathfrak{O}_{\ell} \cong \mathfrak{O}_{j}$. Then

$$
\begin{aligned}
\rho_{i j} & =\sum_{\ell \sim j} c_{i \ell}=\sum_{\ell \sim j} \sum_{r=1}^{H} b_{i r}(m) b_{r \ell}(n) \\
& =\sum_{r=1}^{H} b_{i r}(m)\left(\sum_{\ell \sim j} b_{r \ell}(n)\right)=\sum_{r=1}^{H} b_{i r}(m) \pi_{r j}\left(n^{2}\right) \\
& =\sum_{k=1}^{T} \sum_{r \sim k} b_{i r}(m) \pi_{r j}\left(n^{2}\right)=\sum_{k=1}^{T}\left(\sum_{r \sim k} b_{i r}(m)\right) \pi_{k j}\left(n^{2}\right)
\end{aligned}
$$




$$
=\sum_{k=1}^{T} \pi_{i k}\left(m^{2}\right) \pi_{k j}\left(n^{2}\right), \quad \text { as was to be shown. }
$$

We extend the definition of Anzahlmatrix by setting

$$
P\left(n^{2}\right)=\overline{B(n)} \quad \text { for } n \geq 0 .
$$

Proposition 6. (i) If $(m, n)=1$, then $P\left(m^{2}\right) P\left(n^{2}\right)=P\left(m^{2} n^{2}\right)$.

(ii) If $p \mid d$, then $P\left(p^{2 a}\right)=I$ for all $a \geq 0$.

(iii) If $p \nmid d$ and $0 \leq a \leq b$, then

$$
P\left(p^{2 a}\right) P\left(p^{2 b}\right)=\sum_{k=0}^{a} p^{k} P\left(p^{2(a+b-2 k)}\right) .
$$

Proof. Each assertion is an immediate consequence of the Lemma and the corresponding multiplicative property of the $B(m)$ ([3], p. 138).

\section{§. Shimura's correspondence}

In this section we explicitly determine the effect of Shimura's correspondence on theta series associated to ternary lattices of type I and II. For each such $\theta(z)$ and square-free integer $t \geq 1$ we will express $\theta^{(t)}(z)$ in terms of the theta series associated to the quaternary lattices $\mathfrak{R}_{k \ell}$ defined in $\S 4$. As a simple application, we will also be able to determine the effect on theta series associated to $\delta$-transforms of type I or type II lattices. We first settle the matter of $P^{*}\left(p^{2}\right)=P\left(p^{2}\right)$.

Lemma. Let $p$ be a prime. Then for any $i, j$ we have

$$
\pi_{i j}\left(p^{2}\right) w_{j} 2^{f_{j}}=\pi_{j i}\left(p^{2}\right) w_{i} 2^{f_{i}} .
$$

Proof. Applying a result of Eichler ([1], p. 110, (17.8)) and keeping in mind that his Anzahlmatrices are the transposes of ours, we see that

$$
\pi_{i j}\left(p^{2}\right) v_{j}=\pi_{j i}\left(p^{2}\right) v_{i},
$$

where $v_{k}$ denotes the order of the orthogonal automorphism group of $L_{k}$, $k=1, \cdots, T$. Any orthogonal automorphism of $L_{k}$ is of the form $\xi \mapsto$ $\pm \alpha^{-1} \xi \alpha$ for some $\alpha \in \mathfrak{N}\left(\mathfrak{O}_{k}\right)$. It is easily seen that $\left[\mathfrak{N}\left(\bigcirc_{k}\right): \boldsymbol{Q}^{\times}\right]=w_{k} 2^{f_{k-1}}$ ([7], p. 299). Then $v_{k}=w_{k} 2^{f_{k}}$ and the assertion follows from (42).

Proposition 7. Assume $d$ is odd. Let $L_{1}, \cdots, L_{T}$ represent the isometry classes of type I lattices, so that $L_{1}^{*}, \cdots, L_{T}^{*}$ represent the isometry classes of type II lattices. Let $p$ be a prime and $P\left(p^{2}\right)$, resp. $P^{*}\left(p^{2}\right)$, the associated 
Anzahlmatrix with respect to $L_{1}, \cdots, L_{T}$, resp. $L_{1}^{*}, \cdots, L_{T}^{*}$. Then $P^{*}\left(p^{2}\right)=$ $P\left(p^{2}\right)$.

Proof. Let $\alpha \in \mathfrak{U}^{\times}$. Then

$$
\begin{aligned}
& p \alpha^{-1} L_{j}^{*} \alpha \subset L_{i}^{*} \Leftrightarrow p \alpha^{-1} L_{j}^{\#} \alpha \subset L_{i}^{\#} \\
& \quad \Leftrightarrow p^{-1} \alpha^{-1} L_{j} \alpha \supset L_{i} \Leftrightarrow p \alpha L_{i} \alpha^{-1} \subset L_{j} .
\end{aligned}
$$

Thus $\alpha \mapsto \alpha^{-1}$ gives a one-to-one correspondence between elements $\alpha$ such that $p \alpha^{-1} L_{j}^{*} \alpha \subset L_{i}^{*}$ and elements $\beta$ such that $p \beta^{-1} L_{i} \beta \subset L_{j}$. Furthermore, it is easily seen that $p \alpha^{-1} L_{j}^{*} \alpha=p L_{i}^{*} \Leftrightarrow p \alpha L_{i} \alpha^{-1}=p L_{j}$. The number of $\alpha$ 's is $\pi_{i j}^{*}\left(p^{2}\right) w_{j} 2^{f_{j}}$, while the number of $\beta^{\prime} \mathrm{s}$ is $\pi_{j i}\left(p^{2}\right) w_{i} 2^{f_{i}}$. Applying the Lemma, we get

$$
\pi_{i j}^{*}\left(p^{2}\right) w_{j} 2^{f_{j}}=\pi_{j i}\left(p^{2}\right) w_{i} 2^{f_{i}}=\pi_{i j}\left(p^{2}\right) w_{j} 2^{f_{j}},
$$

proving the result.

Let $t \geq 1$ be a square-free integer. Following Shimura ([11], p. 458), we define $\chi_{t}$, a character $\bmod N t$, as follows:

$$
\chi_{t}(n)=\left\{\begin{array}{cc}
\left(\frac{-t}{n}\right) & \text { if }(n, N t)=1 \\
0 & \text { otherwise }
\end{array} .\right.
$$

In particular, $\chi_{\iota}(n)=0$ if $n$ is even. Let $L$ be a lattice of type I or II. We set

$$
\theta^{(t)}=\theta^{(t)}(z, L)=\sum_{n=0}^{\infty} A^{(t)}(n) e^{2 \pi i n z},
$$

where $A^{(t)}(n)$ is defined for $n \geq 1$ by

$$
\sum_{n=1}^{\infty} A^{(t)}(n) n^{-s}=\left(\sum_{n=1}^{\infty} a\left(t n^{2}, L\right) n^{-s}\right)\left(\sum_{n=1}^{\infty} \chi_{t}(n) n^{-s}\right)
$$

and $A^{(t)}(0)$ is the unique value which makes $\theta^{(t)}$ a modular form.

Let $L_{1}, \cdots, L_{T}$ represent the isometry classes of type I lattices. If $d$ is odd, let $L_{1}^{*}, \cdots, L_{T}^{*}$ be the corresponding type II lattices. Put

$$
\begin{aligned}
& \theta_{i}=\theta\left(z, L_{i}\right)=\sum_{n=0}^{\infty} a_{i}(n) e^{2 \pi i n z}, \\
& \theta_{i}^{*}=\theta\left(z, L_{i}^{*}\right)=\sum_{n=0}^{\infty} a_{i}^{*}(n) e^{2 \pi i n z}, \quad i=1, \cdots, T .
\end{aligned}
$$


Let $\theta_{G}=\left[\theta_{i}\right], \theta_{G^{*}}=\left[\theta_{i}^{*}\right], a(t)=\left[a_{i}(t)\right], a^{*}(t)=\left[a_{i}^{*}(t)\right]$, all considered as column vectors. Put

$$
\begin{aligned}
\Theta=\Theta(z) & =\sum_{n=0}^{\infty} B(n) e^{2 \pi i n z}, \\
\bar{\Theta} & =\bar{\Theta}(z)=\sum_{n=0}^{\infty} \overline{B(n)} e^{2 \pi i n z} .
\end{aligned}
$$

THEOREM 1. Let $t \geq 1$ be a square-free integer. If $d$ is odd assume that $t \equiv 1,2(\bmod 4)$. Then we have

$$
\theta_{G}^{(t)}=\bar{\Theta} a(t) .
$$

Proof. We must show for each $i$ that

$$
\theta_{i}^{(t)}=\sum_{j=1}^{T} a_{j}(t) \sum_{n=0}^{\infty} \pi_{i j}\left(n^{2}\right) e^{2 \pi i n z} .
$$

Evaluating the $M$-th Fourier coefficient of each side for $M \geq 1$, we must show

$$
\sum_{m \mid M} \chi_{t}(m) a_{i}\left(t M^{2} / m^{2}\right)=\sum_{j=1}^{T} \pi_{i j}\left(M^{2}\right) a_{j}(t)
$$

The proof will be by induction on the number of primes dividing $M$. If $M=1$ the statement is trivial. Suppose (46) is true for $M$ and its divisors, and let $N=M p, p$ a prime. There are two cases: (1) $p \nmid M$ or $p \mid d$, (2) $p \mid M$ and $p \nmid d$.

Case (1). For any $k$ we have

$$
\sum_{i=1}^{T} \pi_{k i}\left(p^{2}\right) \sum_{m \mid M} \chi_{t}(m) a_{i}\left(t M^{2} / m^{2}\right)=\sum_{i=1}^{T} \pi_{k i}\left(p^{2}\right) \sum_{j=1}^{T} \pi_{k i}\left(M^{2}\right) a_{j}(t) .
$$

The right side of $(47)=$

$$
\sum_{j=1}^{T}\left(\sum_{i=1}^{T} \pi_{k i}\left(p^{2}\right) \pi_{i j}\left(M^{2}\right)\right) a_{j}(t)=\sum_{j=1}^{T} \pi_{k j}\left(N^{2}\right) a_{j}(t),
$$

by Proposition 6 (i), (ii). If $p$ is odd or $p=2 \mid d$, the left side of $(47)=$

$$
\begin{aligned}
& \sum_{m \mid M} \chi_{t}(m) \sum_{i=1}^{T} \pi_{k i}\left(p^{2}\right) a_{i}\left(t M^{2} / m^{2}\right) \\
& =\sum_{m \mid M} \chi_{t}(m)\left(a_{k}\left(t M^{2} p^{2} / m^{2}\right)+\chi_{t}(p) a_{k}\left(t M^{2} / m^{2}\right)\right),
\end{aligned}
$$

by Propositions 1 and 2 . We note that (49) is also valid for $p=2$ when $d$ is odd by applying (24) and the fact that $t M^{2} / m^{2}$ is not represented by $L_{i}^{*}$. Then (49) equals 


$$
\begin{gathered}
\sum_{m \mid M} \chi_{t}(m) a_{k}\left(t M^{2} p^{2} / m^{2}\right)+\sum_{m \mid M} \chi_{t}(m p) a_{k}\left(t M^{2} p^{2} / m^{2} p^{2}\right) \\
=\sum_{n \mid N} \chi_{t}(n) a_{k}\left(t N^{2} / n^{2}\right), \text { as required. }
\end{gathered}
$$

Case (2). In this case, applying Proposition 6 (iii), we see that the right side of (47) equals

$$
\begin{aligned}
& \sum_{j=1}^{T}\left(\pi_{k j}\left(N^{2}\right)+p \pi_{k j}\left(M^{2} / p^{2}\right)\right) a_{j}(t) \\
& \quad=\sum_{j=1}^{T} \pi_{k j}\left(N^{2}\right) a_{j}(t)+p \sum_{j=1}^{T} \pi_{k j}\left(M^{2} / p^{2}\right) a_{j}(t) .
\end{aligned}
$$

On the other hand, the left side of (47) equals

$$
\begin{gathered}
\sum_{\substack{m \mid M \\
p \nmid(M / m)}} \chi_{t}(m) \sum_{i=1}^{T} \pi_{k i}\left(p^{2}\right) a_{i}\left(t M^{2} / m^{2}\right)+ \\
\sum_{\substack{m|M \\
p|(M / m)}} \chi_{t}(m) \sum_{i=1}^{T} \pi_{k i}\left(p^{2}\right) a_{i}\left(t M^{2} / m^{2}\right)
\end{gathered}
$$

The evaluation of (51) proceeds exactly as in Case (1) to give

$$
\sum_{\substack{m \mid M \\ p \nmid(M / m)}} \chi_{t}(m) a_{k}\left(t M^{2} p^{2} / m^{2}\right)+\sum_{\substack{m \mid M \\ p \nmid(M / m)}} \chi_{t}(m p) a_{k}\left(t M^{2} p^{2} / m^{2} p^{2}\right) .
$$

After application of Proposition 1 and (25), (52) becomes

$$
\sum_{\substack{m|M \\ p|(M / m)}} \chi_{t}(m) a_{k}\left(t M^{2} p^{2} / m^{2}\right)+p \sum_{\substack{m|M \\ p|(M / m)}} \chi_{t}(m) a_{k}\left(t M^{2} / m^{2} p^{2}\right) .
$$

Adding (53) to (54), we see that the left side of (47) equals

$$
\begin{gathered}
\sum_{n \mid N} \chi_{t}(n) a_{k}\left(t N^{2} / n^{2}\right)+p \sum_{m \mid(M / p)} \chi_{t}(m) a_{k}\left(t M^{2} / p^{2} m^{2}\right) \\
=\sum_{n \mid N} \chi_{t}(n) a_{k}\left(t N^{2} / n^{2}\right)+p \sum_{j=1}^{T} \pi_{k j}\left(M^{2} / p^{2}\right) a_{j}(t),
\end{gathered}
$$

by the inductive hypothesis. By equating (55) with (50), the Theorem is proved.

Assume that $d$ is odd. Let $t \geq 1$ be square-free, $t \equiv 3(\bmod 4)$. Define $\omega_{t}$, a character $\bmod d t$, as follows

$$
\omega_{t}(n)=\left\{\begin{array}{cl}
\left(\frac{-t}{n}\right) & \text { if }(n, d t)=1 \\
0 & \text { otherwise }
\end{array} .\right.
$$

We note that 


$$
\omega_{t}\left(2^{k} n\right)=\left(\frac{-t}{2}\right)^{k} \chi_{t}(n) \quad \text { if } n \text { is odd }
$$

Let $L^{*}$ be a lattice of type II. We define a "modified" Shimura lifting $\left(\theta^{*}\right)^{[t]}$ by

$$
\left(\theta^{*}\right)^{[t]}=\sum_{n=0}^{\infty} A^{[t]}(n) e^{2 \pi i n z},
$$

where $A^{[t]}(0)=A^{(t)}(0)$ and

$$
\sum_{n=1}^{\infty} A^{[t]}(n) n^{-s}=\left(\sum_{n=1}^{\infty} a\left(t n^{2}, L^{*}\right) n^{-s}\right)\left(\sum_{n=1}^{\infty} \omega_{t}(n) n^{-s}\right) .
$$

Replacing $\chi_{t}$ by $\omega_{t}$ and applying Proposition 4, we can proceed as in the proof of Theorem 1 to obtain:

Lemma. Assume $d$ is odd. Let $t \geq 1$ be a square-free integer, $t \equiv 3$ $(\bmod 4)$. Then

$$
\theta_{G^{*}}^{[t]}=\bar{\Theta} a^{*}(t) .
$$

Theorem 2. Assume $d$ is odd. Let $t \geq 1$ be a square-free integer, $t \equiv 3$ $(\bmod 4)$. Then

$$
\theta_{G^{*}}^{(t)}=\left(\bar{\Theta}(z)-\left(\frac{-t}{2}\right) \bar{\Theta}(2 z)\right) a^{*}(t)
$$

Proof. By (56), we have $\sum \omega_{t}(n) n^{-s}=\left(\sum \chi_{t}(n) n^{-s}\right)\left(1-(-t / 2) 2^{-s}\right)^{-1}$. It follows that

$$
\begin{array}{r}
\sum_{n=1}^{\infty} A^{(t)}(n) n^{-s}=\left(\sum_{n=1}^{\infty} A^{[t]}(n) n^{-s}\right)\left(1-\left(\frac{-t}{2}\right) 2^{-s}\right) \\
=\sum_{n=1}^{\infty} A^{[t]}(n) n^{-s}-\left(\frac{-t}{2}\right) \sum_{n=1}^{\infty} A^{[t]}(n)(2 n)^{-s} .
\end{array}
$$

Hence $\left(\theta_{i}^{*}\right)^{(t)}(z)=\left(\theta_{i}^{*}\right)^{[t]}(z)-(-t / 2)\left(\theta_{i}^{*}\right)(2 z)$. The proof is completed by applying the Lemma.

If $f(z)$ is an integral modular form of weight two and level $d$, then $f(2 z)$ is an integral modular form of weight two and level $2 d$. In particular, Theorem 2 shows that liftings $\left(\theta_{i}^{*}\right)^{(t)}$ are of level $2 d$ when $t \equiv 3$ ( $\bmod 4)$. This is to be contrasted with the liftings $\theta_{i}^{(t)}$ of Theorem 1 , which are of level $d$. On the space of integral modular forms of weight two and level $2 d$ we have operators $U(p)$ defined for primes $p \mid 2 d$ as follows: 


$$
\text { If } f(z)=\sum_{n=0}^{\infty} a(n) e^{2 \pi i n z} \text {, then }(f \mid U(p))(z)=\sum_{n=0}^{\infty} a(p n) e^{2 \pi i n z} .
$$

From the definition (43), (44) of $\theta^{(t)}$, it is clear that

$$
\left(\theta \mid T_{3, x}^{N}\left(p^{2}\right)\right)^{(t)}=\theta^{(t)} \mid U(p) \quad \text { for } p \mid 2 d .
$$

In addition, for $(n, d)=1$ we have Hecke operators $T(n)$ defined on the forms of level $d$. If $f(z)$ is an integral modular form of weight two and level $d$, then

$$
(f \mid T(2))(z)=(f \mid U(2))(z)+2 f(2 z) .
$$

The Hecke operators $T(n)$ satisfy the following commutation relation ([4], p. 166):

$$
(\Theta \mid T(n))(z)=B(n) \Theta(z)
$$

It follows that

$$
\bar{\Theta} \mid T(n)=\overline{(\Theta \mid T(n))}=\overline{(B(n) \Theta(z))}=\overline{B(n)} \bar{\Theta}(z)
$$

for $(n, d)=1$. We are in a position now to evaluate the liftings in the remaining cases.

Theorem 3. Assume $d$ is odd. Let $t \geq 1$ be a square-free integer.

(i) If $t \equiv 1,2(\bmod 4)$, then

$$
\theta_{G^{*}}^{(t)}=\bar{\Theta}(2 z) a(t)
$$

(ii) If $t \equiv 3(\bmod 4)$, then

$$
\theta_{G^{*}}^{(t)}=\bar{\Theta}(z) a(t)-2 \bar{\Theta}(2 z) a^{*}(t) .
$$

Proof. First suppose $t \equiv 1,2(\bmod 4)$. Applying the Corollary to Proposition 4 , we have

$$
\begin{aligned}
\theta_{G^{*}}^{(t)} & \left.=\frac{1}{2} \overline{(B(2)} \theta_{G}-\theta_{G} \mid T_{3, \chi}^{N}(4)\right)^{(t)} \\
& \left.=\frac{1}{2} \overline{(B(2)} \theta_{G}^{(t)}-\theta_{G}^{(t)} \mid U(2)\right)=\theta_{G}^{(t)}(2 z),
\end{aligned}
$$

by (59), (62) and (60). The result then follows from Theorem 1.

Now suppose $t \equiv 3(\bmod 4)$. Applying Proposition 3 and Theorem 2, we obtain

$$
\begin{aligned}
\theta_{G}^{(t)} & =\left(\theta_{G^{*}} \mid T_{3, x}^{N}(4)\right)^{(t)}=\theta_{G^{*}}^{(t)} \mid U(2) \\
& =\left(\bar{\Theta}(z)-\left(\frac{-t}{2}\right) \bar{\Theta}(2 z)\right) \mid U(2) \cdot a^{*}(t)=\left(\bar{\Theta} \mid U(2)-\left(\frac{-t}{2}\right) \bar{\Theta}\right) a^{*}(t) .
\end{aligned}
$$


Using (60), (62) and Proposition 4, we see that (63) equals

$$
\begin{aligned}
(\bar{\Theta}(z) & \left.\overline{B(2)}-2 \bar{\Theta}(2 z)-\left(\frac{-t}{2}\right) \bar{\Theta}(z)\right) a^{*}(t) \\
= & \bar{\Theta}(z)\left(P(4) a^{*}(t)\right)-2 \bar{\Theta}(2 z) a^{*}(t)-\left(\frac{-t}{2}\right) \bar{\Theta}(z) a^{*}(t) \\
= & \bar{\Theta}(z)\left(a^{*}(4 t)+\left(\frac{-t}{2}\right) a^{*}(t)\right)-2 \bar{\Theta}(2 z) a^{*}(t)-\left(\frac{-t}{2}\right) \bar{\Theta}(z) a^{*}(t) \\
= & \bar{\Theta}(z) a(t)-2 \bar{\Theta}(2 z) a^{*}(t)
\end{aligned}
$$

as asserted.

Let $\delta \mid d$, where $d$ can be even or odd. Suppose $M$ is similar to $L^{(\delta)}$, where $L$ is of type I or type II. Let $t \geq 1$ be square-free, $\delta_{1}=\delta /(t, \delta)$. Then

$$
a\left(t n^{2}, M\right)=a\left(t \delta n^{2}, L\right)=a\left(t \delta_{1} n^{2}, L\right),
$$

by the proof of Proposition 2. Let $\theta=\theta(z, M)$. To determine $\theta^{(t)}$ in this case, one takes the character $\chi_{t}$ defined by:

$$
\chi_{t}(n)=\left\{\begin{array}{cl}
\left(\frac{-t \delta_{1}}{n}\right) & \text { for }(n, N t)=1 \\
0 & \text { otherwise }
\end{array} .\right.
$$

It is clear that Theorems $1,2,3$ carry over to $\delta$-transforms of type I or type II lattices, with the congruence conditions on $t \delta_{1}$ instead of $t$ and with the quadratic symbol $\left(-t \delta_{1} / 2\right)$ instead of $(-t / 2)$. More precisely, let $L_{1}, \cdots, L_{T}$ represent the similitude classes in $G$. Let $M_{i}=L_{i}^{(\delta)}, i=1, \cdots, T$. Let $\Gamma$ denote the similitude genus containing the $M_{i}$. If $d$ is odd, let $\Gamma^{*}$ denote the similitude genus containing the $M_{i}^{*}$. Let $\theta_{\Gamma}=\left[\theta\left(z, M_{i}\right)\right], \theta_{\Gamma^{*}}=$ $\left[\theta\left(z, M_{i}^{*}\right)\right], a(t)=\left[a\left(t, M_{i}\right)\right], a^{*}(t)=\left[a\left(t, M_{i}^{*}\right)\right]$. Then we have:

Corollary. Let $\delta, \theta_{\Gamma}, \theta_{\Gamma^{*}}, a(t), a^{*}(t)$ be as just defined.

(i) Suppose $d$ is even, or $d$ is odd and $t \delta \equiv 1,2(\bmod 4)$. Then

$$
\begin{aligned}
& \theta_{\Gamma}^{(t)}=\bar{\Theta} a(t), \\
& \theta_{\Gamma^{*}}^{(t)}=\bar{\Theta}(2 z) a(t) \quad(\text { if } d \text { is odd }) .
\end{aligned}
$$

(ii) Suppose $d$ is odd, to $\equiv 3(\bmod 4)$. Then

$$
\begin{aligned}
& \theta_{\Gamma}^{(t)}=\bar{\Theta}(z) a(t)-2 \bar{\Theta}(2 z) a^{*}(t) \\
& \theta_{\Gamma^{*}}^{(t)}=\left(\bar{\Theta}(z)-\left(\frac{-t \delta}{2}\right) \bar{\Theta}(2 z)\right) a^{*}(t) .
\end{aligned}
$$




\section{§6. Concluding Remarks}

The dependence on $t$ of Shimura's correspondence $\varphi \mapsto \varphi^{(t)}$ can be circumvented if $\varphi$ is a cusp form which is a common eigenfunction for all Hecke operators $T_{3, x}^{N}\left(p^{2}\right)$. In that case $\varphi^{(t)}=a(t) \cdot f$, where $a(t)$ is the $t$-th Fourier coefficient of $\varphi$ and $f$ is independent of $t$ ([11], p. 453). It is natural to call $f$ the "Shimura lifting of $\varphi$." The theta series we have considered are neither cusp forms nor, in general, Hecke eigenfunctions. In this section we will consider the question of the existence of Hecke eigenfunctions in the space spanned by these theta series. In addition, we will generalize a result of Niwa's on the image of Shimura's correspondence when $d$ is an odd prime.

As before, let $\theta_{1}, \cdots, \theta_{T}$ be the theta series associated to the isometry classes of type I lattices. If $d$ is odd let $\theta_{1}^{*}, \cdots, \theta_{T}^{*}$ be the corresponding theta series associated to type II classes. Let $\Omega$ be the $C$-linear span of $\theta_{1}, \cdots, \theta_{T}, \Omega^{*}$ the $C$-linear span of $\theta_{1}^{*}, \cdots, \theta_{T}^{*}$. The basic problem we must address is whether $\Omega$, resp. $\Omega+\Omega^{*}$, has a basis of common eigenfunctions for all $T_{3, \chi}^{N}\left(p^{2}\right)$ when $d$ is even, resp. $d$ is odd.

Putting $\mathscr{V}=\left[v_{i} \delta_{i j}\right]$, where the $v_{i}$ are as in (42), we have

$$
\overline{B(p)} \mathscr{V}=P\left(p^{2}\right) \mathscr{V}=\mathscr{V} P\left(p^{2}\right)^{t}=\mathscr{V} \overline{B(p)}
$$

for all primes $p$. It follows that

$$
\overline{B(n)} \mathscr{V}=\mathscr{V} \overline{B(n)} \quad \text { for all } n \geq 0 .
$$

Then $R(n)=\sqrt{\mathscr{V}}^{-1} B(n) \sqrt{\mathscr{V}}$ is symmetric for all $n \geq 0$. Since $\{R(n)\}$ is a commuting family of symmetric real matrices, there exists an invertible real matrix $C$ such that $C \overline{B(n)} C^{-1}$ is a diagonal real matrix for all $n \geq 0$ (cf. [1], p. 111). Let us write

$$
C \overline{B(n)} C^{-1}=\left[\begin{array}{lll}
\beta_{1}(n) & & \\
& \ddots & \\
& & \beta_{T}(n)
\end{array}\right],
$$

and put $f_{i}(z)=\sum_{n=0}^{\infty} \beta_{i}(n) e^{2 \pi i n z}, i=1, \cdots, T$. Then

$$
C \bar{\Theta} C^{-1}=\left[\begin{array}{lll}
f_{1} & & \\
& \ddots & \\
& & f_{T}
\end{array}\right] .
$$

From (62) it follows that $f_{i} \mid T(n)=\beta_{i}(n) f_{i}$ for $(n, d)=1$. For $p \mid d$ it is clear that 


$$
\bar{\Theta} \mid U(p)=\overline{\Theta \mid U(p)}=\overline{\Theta B(p)}=\bar{\Theta} \overline{B(p)}=\bar{\Theta},
$$

from which it follows that $f_{i} \mid U(p)=f_{i}$ for all $p \mid d$. Hence each $f_{i}(z)$ is a common eigenfunction for all $T(n),(n, d)=1$, and $U(p), p \mid d$, and we have:

$$
\sum_{n=1}^{\infty} \beta_{i}(n) n^{-s}=\prod_{p \nmid d}\left(1-\beta_{i}(p) p^{-s}+p^{1-2 s}\right)^{-1} \prod_{p \backslash d}\left(1-p^{-s}\right)^{-1} .
$$

Suppose first that $d$ is even. Let $\varphi=\left[\varphi_{i}(z)\right]$ be defined by $\varphi=C \theta_{G}$. By Propositions 1 and $2, \theta_{G} \mid T_{3, x}^{N}\left(p^{2}\right)=\overline{B(p)} \theta_{G}$ for all $p$. It follows that $\varphi \mid T_{3, \chi}^{N}\left(p^{2}\right)=C \overline{B(p)} C^{-1} \varphi$, so that

$$
\varphi_{i} \mid T_{3, x}^{N}\left(p^{2}\right)=\beta_{i}(p) \varphi_{i} \quad \text { for all } p .
$$

In particular, some subset of $\left\{\varphi_{i}\right\}$ provides a basis of $\Omega$ of common eigenfunctions for all the $T_{3, x}^{N}\left(p^{2}\right)$. Furthermore, if $\varphi_{i}(z)$ is a cusp form, then its Shimura lifting must be $f_{i}(z)$. We put

$$
E(z)=\sum_{i=1}^{T} v_{i}^{-1} \theta_{i}(z) .
$$

Then $E \mid T_{3, \chi}^{N}\left(p^{2}\right)=E$ for $p \mid d$, and for $p \nmid d$ we have

$$
\begin{aligned}
E \mid T_{3, x}^{N}\left(p^{2}\right) & =\sum_{i} v_{i}^{-1} \sum_{j} \pi_{i j}\left(p^{2}\right) \theta_{j}=\sum_{j} \sum_{i} v_{i}^{-1} \pi_{i j}\left(p^{2}\right) \theta_{j} \\
& =\sum_{j} \sum_{i} v_{j}^{-1} \pi_{j i}\left(p^{2}\right) \theta_{j}=\sum_{j}(p+1) v_{j}^{-1} \theta_{j}=(p+1) E,
\end{aligned}
$$

by (6) of $\S 2$. We conclude that $E(z)$ lifts to the Eisenstein series

$$
E_{2}(z)=\frac{\phi(d)}{24}+\sum_{n=1}^{\infty} \sum_{\substack{m, n \\(m, d)=1}} m e^{2 \pi i n z} .
$$

Since $\theta_{i}-\theta_{j}$ is a cusp form for all $i, j, \theta_{i}-M^{-1} E$ is a cusp form for each $i$, where $M=\sum_{j} v_{j}^{-1}$. It follows that the subspace of cusp forms in $\Omega$ is of codimension one. Without loss of generality, we may assume $\varphi_{1}=E$, $f_{1}=E_{2}$. Then any $\varphi_{i} \neq E$ must be a cusp form.

Now suppose $d$ is odd. Let $C, \varphi$ be as above, and put $\varphi^{*}=\left[\varphi_{i}^{*}(z)\right]$ $=C \theta_{G^{*}}$. Then for each $i$ we have

$$
\left[\begin{array}{c}
\varphi_{i} \\
\varphi_{i}^{*}
\end{array}\right] \mid T_{3, x}^{N}\left(p^{2}\right)=\beta_{i}(p)\left[\begin{array}{c}
\varphi_{i} \\
\varphi_{i}^{*}
\end{array}\right] \quad \text { for } p \neq 2
$$

Furthermore, by Proposition 3, and the Corollary to Proposition 4, we have 


$$
\begin{aligned}
& {\left[\begin{array}{l}
\varphi \\
\varphi^{*}
\end{array}\right] \mid T_{3, \bar{x}}^{N *}(4)=\left[\begin{array}{cc}
C & 0 \\
0 & C
\end{array}\right]\left[\begin{array}{cc}
B(2) & -2 I \\
I & 0
\end{array}\right]\left[\begin{array}{cc}
C & 0 \\
0 & C
\end{array}\right]^{-1}\left[\begin{array}{l}
\varphi \\
\varphi^{*}
\end{array}\right]}
\end{aligned}
$$

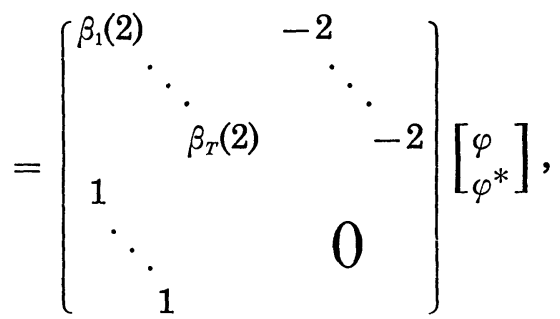

so that

$$
\left[\begin{array}{c}
\varphi_{i} \\
\varphi_{i}^{*}
\end{array}\right] \mid T_{3, \alpha}^{N}(4)=\left[\begin{array}{cr}
\beta_{i}(2) & -2 \\
1 & 0
\end{array}\right]\left[\begin{array}{c}
\varphi_{i} \\
\varphi_{i}^{*}
\end{array}\right], \quad i=1, \cdots, T
$$

Putting $g_{i}(z)=f_{i}(2 z)$, we also have:

$$
\left[\begin{array}{l}
f_{i} \\
g_{i}
\end{array}\right] \mid U(2)=\left[\begin{array}{cr}
\beta_{i}(2) & -2 \\
1 & 0
\end{array}\right]\left[\begin{array}{c}
f_{i} \\
g_{i}
\end{array}\right]
$$

The relation (71) is a special case of the following general phenomenon. Let $f$ be an integral modular form of (even) weight $k$ and level $N$. Suppose $f$ is an eigenfunction for a Hecke operator $T(p)$, where $p$ is a prime, $p \nmid N$. Let $\alpha(p)$ be the eigenvalue and put $g(z)=f(p z)$. Then we have:

$$
\left[\begin{array}{l}
f \\
g
\end{array}\right] \mid U(p)=\left[\begin{array}{cc}
\alpha(p) & -p^{k-1} \\
1 & 0
\end{array}\right]\left[\begin{array}{l}
f \\
g
\end{array}\right]
$$

Since $f, g$ are linearly independent, we see that $U(p)$ is diagonalizable on the span of $f, g \Leftrightarrow X^{2}-\alpha(p) X+p^{k-1}$ has two distinct roots. If $f$ is a cusp form, then Deligne's proof of the Petersson conjecture shows that $\alpha(p)^{2} \leq$ $4 p^{k-1}$ (when $k=2$ this was first proved by Eichler [2]). We conclude that $U(p)$ is diagonalizable on the span of $f, g \Leftrightarrow d(p)^{2}<4 p^{k-1}$. In particular, for square-free level $M$, the diagonalizability of the operators $U(p)$ on old forms of level $M$ is equivalent to this "strong" form of Petersson's conjecture for all new forms of level a proper divisor of $M$.

Returning to our situation, we see that if $\varphi_{i}, \varphi_{i}^{*}$ are cusp forms and $\psi_{i}$ is an eigenfunction for $T_{3 . \chi}^{N}(4)$ in the span of $\varphi_{i}, \varphi_{i}^{*}$, then the lifting of $\psi_{i}$ will have a Dirichlet series with the following Euler product:

$$
\left(1-\sigma_{i}(2) 2^{-s}\right)^{-1} \prod_{p \nmid 2 d}\left(1-\beta_{i}(p) p^{-s}+p^{1-2 s}\right)^{-1} \prod_{p \mid d}\left(1-p^{-s}\right)^{-1},
$$

where 


$$
\left(1-\sigma_{i}(2) 2^{-s}\right)\left(1-\overline{\sigma_{i}(2)} 2^{-s}\right)=\left(1-\beta_{i}(2) 2^{-s}+2^{1-2 s}\right) .
$$

In particular, the lifting of $\psi_{i}$ will be an old form of level $2 d$ which is an eigenfunction for all $U(p), p \mid 2 d$. Moreover, $\Omega+\Omega^{*}$ has a basis of common eigenfunctions for all $T_{3, x}^{N}\left(p^{2}\right)$ unless, for some $i, \varphi_{i}, \varphi_{i}^{*}$ are linearly independent and $\beta_{i}(2)^{2}=8$, in which case $\sigma_{i}(2)=\overline{\sigma_{i}(2)}= \pm \sqrt{2}$. Looking at the tables of Wada [12], we see that $\beta_{i}(2)^{2}<8$ if $d$ is any odd prime $<250$.

For $i=1$ we have $\varphi_{1}=E, \varphi_{1}^{*}=E^{*}=\sum v_{i}^{-1} \theta_{i}^{*}, \beta_{i}(2)=3$. Then $E-$ $2 E^{*}, E-E^{*}$ are eigenfunctions for $T_{3, x}^{N}(4)$ with eigenvalues 1 , 2, resp. The corresponding liftings are $E_{2}(z)-2 E_{2}(2 z), E_{2}(z)-E_{2}(2 z)$, resp., where $E_{2}(z)$ is as in (69).

We conclude with a discussion of the image of Shimura's correspondence in the special case $d=p$, an odd prime. For $i, k \leq H, j \leq T$, let

$$
\begin{aligned}
& \theta_{i k}(z)=\sum_{n=0}^{\infty} b_{i k}(n) e^{2 \pi i n z}, \\
& \bar{\theta}_{i j}(z)=\sum_{k \sim j} \theta_{i k}(z) .
\end{aligned}
$$

Using the notation of $\S 4, \theta_{i j}(z)=w_{j}^{-1} \theta\left(z, f_{j i}\right)$ is the theta series associated to $\mathfrak{R}_{j i}$. It is easily seen that $\mathfrak{D}_{j}^{\#}=\mathfrak{P}_{j}^{-1}$, where $\mathfrak{P}_{j}$ is the unique two-sided ideal of $\mathfrak{D}_{j}$ with norm $p$. It follows that

$$
\mathfrak{R}_{j i}^{\#}=N\left(\mathfrak{R}_{i j}\right) \mathfrak{P}_{j}^{-1} \mathfrak{\Omega}_{j i} .
$$

Then $f_{j j}$ represents $p \Leftrightarrow \Re_{j}$ is principal $\Leftrightarrow \bar{\theta}_{i j}=\theta_{i j} \Leftrightarrow f_{j i}$ is equivalent to $f_{j i}^{\#}$. If $\mathfrak{P}_{j}$ is not principal, then

$$
\bar{\theta}_{i j}(z)=w_{j}^{-1}\left(\theta\left(z, f_{j i}\right)+\theta\left(z, f_{j i}^{\sharp}\right)\right) .
$$

Let $\mathfrak{M}$ denote the space of modular forms of weight 2 and level $p$. We have an operator $W(p)$ on $\mathfrak{M}$ defined by

$$
(f \mid W(p))(z)=\left(f \mid\left[\begin{array}{rr}
0 & -1 \\
p & 0
\end{array}\right]\right)(z)=p^{-1} z^{-2} f(-1 / p z)
$$

Let $\mathfrak{M}^{-}$denote the subspace of $\mathfrak{M}$ of all $f$ such that $f \mid W(p)=-f$. The inversion formula for theta series (cf. [1], p. 136, (20.6)) implies that $\bar{\theta}_{i j} \in$ $\mathfrak{M}^{-}$for all $i, j$. Let $g, g^{*}$ denote the genus of $\Gamma_{0}(p),\left\langle\Gamma_{0}(p),\left[\begin{array}{rr}0 & -1 \\ p & 0\end{array}\right]\right\rangle$, resp. Then $g=H-1, \operatorname{dim} \mathfrak{M}^{-}=H-g^{*}$. Comparing the classical formula of Fricke for $g-2 g^{*}$ ([14], p. 408) with the formula for $T$ ([3], p. 147), we see that 
$\operatorname{dim} \mathfrak{M}^{-}=T$.

Since the level is prime, $\mathfrak{M}^{-}$has a basis consisting of $E_{2}$ and newforms of level $p$. Let $\mathfrak{M}_{\text {old }}^{-}$denote the span of $E_{2}(z), E_{2}(2 z)$ and the oldforms of level $2 p$ coming from $\mathfrak{M}^{-}$. It is clear that $\operatorname{dim} \mathfrak{M}_{\text {old }}^{-}=2 T$. As a direct consequence of Theorems 1-3 we have

Proposition 8. Let $d=p$, an odd prime, $t \geq 1 a$ square free integer.

(i) If $L$ is a lattice of type I and $t \equiv 1,2(\bmod 4)$, then $\theta^{(t)}(z, L) \in \mathfrak{M}$ -

(ii) If $L$ is of type II, or if $L$ is of type I and $t \equiv 3(\bmod 4)$, then $\theta^{(t)}(z, L) \in \mathfrak{M}_{\text {old }}^{-}$.

Remarks 1. Using the Corollary at the end of $\S 5$, we see that the same results hold for $p$-transforms after replacing $t$ by $t p$ in the congruences.

2. The case $t \equiv 2(\bmod 4)$ was first proved by Niwa in a letter to Shimura in 1975.

3. The fact that $\operatorname{dim} \mathfrak{M}^{-}=T, \operatorname{dim} \mathfrak{M}_{\text {old }}^{-}=2 T$ suggests that the theta series $\theta_{i}, \theta_{2}^{*}$ are linearly independent and the Shimura lifting (or $\theta \mapsto \theta^{(t)}$ for certain $t$ ) is a bijection.

\section{REFERENCES}

[1] M. Eichler, Quadratische Formen und orthogonale Gruppen, Springer-Verlag, Berlin-Göttingen-Heidelberg, 1952.

[2] —-, Quaternäre quadratische Formen und die Riemannsche Vermutung für die Kongruenzzetafunktion, Archiv der Math. 5 (1954), 355-366.

[ 3 ] _ _ Zur Zahlentheorie der Quaternionen-Algebren, J. Reine Angew. Math. 195 (1955), 127-151.

[ 4 ] — U Über die Darstellbarkeit von Modulformen durch Thetareihen, J. Reine Angew. Math. 195 (1955), 156-171.

[5] M. Kneser, Witts Satz für quadratische Formen über lokalen Ringen, Nachr. Akad. Wiss. Göttingen Math.-Phys. Kl. II 1972, 195-203.

[6] S. Niwa, Modular forms of half integral weight and the integral of certain thetafunctions, Nagoya Math. J. 56 (1974), 147-161.

[ 7 ] P. Ponomarev, Class numbers of definite quaternary forms with square discriminant, J. No. Theory 6 (1974), 291-317.

[ 8 ] - Arithmetic of quaternary quadratic forms, Acta Arith. 29 (1976), 1-48.

[9] - Ternary quadratic forms and an explicit quaternary correspondence, Proceedings of the Conference on Quadratic Forms, Queen's Papers in Pure and Applied Mathematics, No. 46 (1977), 582-594.

[10] S. Rallis, The Eichler commutation relation and the continuous spectrum of the Weil representation, Proceedings of the Conference on Non Commutative Harmonic Analysis, Marseille-Luminy, June 1978, Springer-Verlag (to appear).

[11] G. Shimura, On modular forms of half integral weight, Ann. of Math. 97 (1973), $440-481$. 
[12] H. Wada, A table of Hecke operators II, Proc. Japan Acad. 49 (1973), 380-384.

[13] G. L. Watson, Transformations of a quadratic form which do not increase the class-number, Proc. London Math. Soc. (3) 12 (1962), 577-587.

[14] M. Yamauchi, On the traces of Hecke operators for a normalizer of $\Gamma_{0}(\mathrm{~N})$, J. Math. Kyoto Univ. 13 (1973), 403-411.

Department of Mathematics

The Ohio State University

Columbus. Ohio 43210 